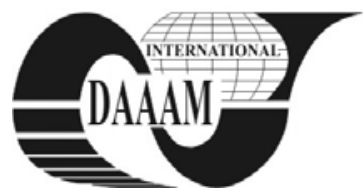

\title{
ADAPTIVE ENVIRONMENT FOR DEVELOPING AND PROGRAMMING OF MOBILE ROBOTS
}

\author{
PRYANICHNIKOV, V[alentin] E.; ANDREEV, V[ictor] P[avlovich]; IVCHENKO, V[alery]; \\ KUVSHINOV, S[ergey] \& PRYSEV, E[vgeniy] A[leksandrovich]
}

\begin{abstract}
This study considers the creation of a program complex on the basis of a virtual machine with the operating system UNIX pre-installed and a free software suite for elaborating and programming applications for control systems of mobile technological robots. Being a three-level software stack (virtual machine, operating system, and development environment), the program complex is designed for students to be used at laboratory classes of programming for AMUR mobile technological robots within the course offered to universities, and scientific-educational centers of the Russian Academy of Sciences. This study analyses the existing program solutions at each level, sets criteria for software selection (including the account for preparation and skills of students) and gives recommendations on the selection of specific software according to the original program.

Key words: educational and scientific stands, mobile robots, mechatronics, sensorics, computer vision systems
\end{abstract}

\section{INTRODUCTION}

Traditional training of specialists in the integration of complex technical systems with the help of conventional higher education methods is hugely limited because students lack experience in building hardware and software systems supporting the interaction between the human-computer system and real objects of control. We managed to implement a new model of the educational process: student tasks were treated as scientific research to be conducted yearly and the results obtained for each topic became the basis for new topics of research to be conducted by another group. Such a "recursive" method of research made it possible to achieve a suitable quality of research with a successful methodical effect. Actual research in practice yielded a technology of collective development of models and their implementation into the control system for real mobile robots (MR).

If confined merely to mathematical models, the perception of problems by students turned out to be incomplete. In view of this, we developed a system of mechatronics tools and elements as educational and scientific stands (mobile robots) designed for practical study, design, and testing of complex technical systems as well as for performing demonstration classes in the field of sensorics and intelligent systems. The stands are suitable for testing and correcting students' mistakes, which are "materialized" in the incorrect robot operation, allowing one to promptly see the cause and cost of errors.

\section{PROBLEMS OF EDUCATIONAL STANDS}

Educational and scientific stands allow for a pictorial demonstration and design of complex control systems for mechatronics elements on the basis of analysis of the sensorrecorded situation. This helps understand the nature of human interaction with complex technical systems. The simulation of the interrelation between the elements of technical systems and the use of individual and collective mapping enables students to form a comprehensive view on the design, analysis and synthesis, selection of criteria, and system evaluation. This is achieved through the use of communication, interactive tools working in conjunction with video cameras. The quality of results is satisfactory due to the joint efforts of an educational institution (the Institute of New Educational Technologies and Informatics of the Russian State University for the Humanities), the Institute of Applied Mathematics of the Russian Academy of Sciences and the International Laboratory "Sensor".

The question arises: "Why it is the robotic and mechatronic systems that are the best data?" The answer is:

1.Real mobile robots are psychologically easily projected on humans - each student feels himself an expert in this field.

2.The universal computerization, including in the humanitarian field, makes it necessary to perform in-depth training in the methods for building intelligent software, which constitute one of the basic problems of robotics.

3.Complex problems in mechatronics imply principally a creative thinking without divestment or distancing from most topical problems of modern production.

The main educational activities are carried out at the Institute of New Educational Technologies and Informatics of the Russian State University for the Humanities (in the Laboratory of problems of informatics, mechatronics, and sensorics) as well as in the Moscow State University of Equipment design and informatics. The education process is characterized by active involvement of students and postgraduates into the development and creation of units for prospective special mobile caterpillar robots and simulators. This will enable the educational problems and strong scientific projects to be joined into a unified research process.

One can distinguish the following two stages:

1. Design of the module architecture with an onboard computer, microprocessor control units, communication with intelligent sensors, surveillance-scanning devices, power control switches, ultrasonic and TV sensors, and software and microprogram support for navigation, object identification, and management problems (Pryanichnikov et al., 2009);

2. Implementation of AMUR autonomous mobile educational robots, which are used in the Far Eastern National Technological University and the Institute of New Educational Technologies and Informatics together with Festo stands.

\section{TECHNICAL SUPPORT OF GROUP CONTROL}

The problems of efficient information and group control arise in education and in eliminating the consequences of emergency situations. The paper (Andreev et al., 2010) presents our results on the creation of a multi-element group of mobile robots operating as a unified interrelated system capable of operating under combat conditions, including the elimination of heavy disasters. Similar problems are posed in the construction of robotic systems for space: development of methods for group and remote control of autonomous systems based on network technologies; self-diagnostics and self-learning; and 
organization of the human--machine interface (Kuvshinov et al., 2006). The information support of these activities is a key problem that can be efficiently solved through the creation of a unified situational center and relevant software and hardware tools for information analysis and management support.

This formulation of the problem leads to the need for a multilevel system of formation and exchange of information and control data streams. In turn, this necessitates the construction of a mechanism that can treat the interaction of a group of mobile robots and control stations served concurrently by several operators as well as a situational center as the top of hierarchy. A prerequisite of this interaction is the organization of communication channels allowing the unified command center of situation control to be allocated many kilometers away from the work area. It should also be taken into account that one normally employs MRs that should not be limited in the freedom of movement. In addition, the bandwidth of communication channels must be able to ensure the transfer of multi-stream video from computer vision systems of mobile robots to both control stations and the situation center. This problem we solved with the help or network technologies; here, it is important that the elements of the complex turn out to be mobile nodes of a local area network (LAN).

In solving these problems, our experience in the development of a virtual distributed laboratory (Andreev et al., 2009) is useful. This laboratory is designed for remote communication, including training of professionals. The use of network technologies made it possible to combine multiple MR, remote "satellites"-repeaters and remote desks into a single computer network. The protection from unauthorized access was organized through VPN-channels, which were combined into a single network of mobile robots located at the Institute of New Educational Technologies and Informatics, in the building of the Institute of Applied Mathematics of the Russian Academy of Sciences in Moscow, and in the Institute for Automation and Control Processes of the Far East Division of the Russian Academy of Sciences in Vladivostok (IACP).

The investigations performed on this structure made it possible to formulate the principles of construction and operation of educational and research situational centers for the Federal Agency of Atomic Energy and the Russian Ministry for Civil Defense, Emergencies, and Elimination of Consequences of Natural Disasters, which have widely used various robotic systems. We have developed software that allows one not only to remotely watch the "scene", but also to remotely control these robots on the basis of a technology that avoids irregularities and delays in communication channels. The computer network is based on the TCP/IP stack of protocols because this is a currently widespread practice. These kinds of networks have multiple technical implementations, which allow different software and hardware units of all elements of the complex to be combined into a single network. Then, the problem is reduced to the creation of a LAN whose nodes are digital IP-cameras and computing devices on-board a mobile robot, installed on remote modules and repeaters, on the one hand, and on a computer on the operator desk and computers of the situational center, on the other hand; each with their own IP-addresses of a single network.

The studies aimed at the creation of a virtual distributed laboratory resulted in a mechanism of control for multiple mobile robots simultaneously. For this case, we developed a three-level control system. The first level involves just the mobile robots with sensors (ultrasonic, computer vision systems, etc.) and actuators. The second level involves autonomous control systems for individual robots and mobile robot operators. The link between robots was organized through a Wi-Fi digital radiochannel. The third level involves systems of top-level rules, controlled and modified by the commander, who coordinate the operators as well. In this structure, the commander sees the whole scene and receives video data from the computer vision system of all units that are engaged in the operation of MR and remote units. This level can be implemented (in constructing a computer network on the basis of TCP/IP protocols) merely by including commander's computer into the network and supply the system components by appropriate software.

When using the above approach, the process of decisionmaking at the level of the situation center using data of mobile robotic systems that are processed by distributed local control centers becomes more efficient and reasonable. The structure makes it possible to organize global network connectivity at any point. This work was partially supported by the Russian Foundation for Basic Research, project no. 10-07- 00612a.

\section{CONCLUSION}

This study resulted in the creation of a cross-platform distribution software of a virtual machine with the UNIX operating system and a set of pre-installed open-source software for application design and programming. The level of the virtual machine is implemented using the Oracle Virtualbox; the level of the operating system is implemented with the help of Ubuntu-11.04-desktop-i386; the development environment is the Eclipse with the installed PyDev plugin and Pythonrpyc and Python-pygame libraries. We have manged to limit the volume of the distribution software to a DVD size (not more than $4 \mathrm{~GB}$ ). This provided students with a common development platform, which allowed them to significantly accelerate the solution of given problems, to improve the portability of codes and complementarity under the conditions of teamwork. The development of AMUR educational and research software for the IAPC and for the modernization of technological MR BROKK-110D, BROKK-330 for the Russian Ministry for Civil Defense, Emergencies, and Elimination of Consequences of Natural Disasters showed that the proposed approach is efficient and reduces the time needed for creation and debugging of program complexes several times as compared to traditional approaches (Pryanichnikov et al., 2009).

\section{REFERENCES}

Andreev V.P, Kirsanov K.B., Levinski B.M., Pryanichnikov V.E. [et al.] (2009). Problems of the construction of a network of Internet labs using autonomous mobile robots AMUR. Information-Measuring and Control Systems, Moscow: radiotekhnika, 2009, vol. 7, No. 6, pp.124-131

Andreev V., Pryanichnikov V., Prysev E. (2010). Multi-access control of distributed mobile robotic systems on the base of networking technologies, Annals of DAAAM for 2010\&Proceedings of the 21st International DAAAM Symposium, ISSN 1726-9679, pp. 15-16, Zadar, Croatia

Katalinic, B., Kordic, V. (2002) Concept of Design and Scheduling of Self-Organizing Complex Flexible Assembly System, Proceedings of the 4th International Workshop on Emergent Synthesis - IWES 02, ISBN 961-4238-49-3, pp. 89-96, March 12-13, 2002, Kobe, Japan

Kuvshinov S.V., Pryanichnikov V.E., Artemenko O.L., Levinski B.M. (2006). Development of the elements of an educational and scientific environment for problems of mechatronics and sensorics. Information-Measuring and Control Systems, No. 1-2, vol. 4, 2006, Moscow: Radiotekhnika, pp. 170-175

Pryanichnikov V., Andreev V., Prysev E. (2009). Computer vision and control for special mobile robots, Annals of DAAAM for 2009 \& Proceedings of the 20th International DAAAM Symposium "Intelligent Manufacturing \& Automation" 25-28th November 2009. ISSN 1726-9679, pp.1857-1858, Vienna, Austria 